\title{
IX. CARBON AND NITROGEN ANALYSES, LEG 42A
}

\author{
W. Sigl, Lehrstuhl für Geologie, Technische Universität München, West Germany
}

Samples were dried at $40^{\circ} \mathrm{C}$, ground and mixed to a homogeneous powder. Subsequently, samples were divided in several parts for different determinations.

One part was analyzed for its total carbon content. A second one was treated with hydrochloric acid and analyzed for residual "organic" carbon. The difference between total and organic carbon, called here "inorganic" carbon, was used for carbonate calculation (Müller, this volume).

Carbon determinations were made using a LECO Carbon Analyzer. Procedure and precision of this method is described in detail by Boyce and Bode (1972). Relative error of own measurements is calculated to about $\pm 1 \%$.

Nitrogen was determined by the standard microKjeldahl method. Mostly sapropel(ic) sediments were analyzed (Sigl et al., this volume). Due to the small amounts of sample material available, the values reported here have a precision of $\pm 3 \%$ only. In sediments with low nitrogen content $(<0.1 \%)$, the relative analytical error may increase to $\pm 6 \%$. By the Kjeldahl method, the content of total nitrogen is measured. This includes organic nitrogen and so-called fixed and ex- changeable ammonia nitrogen (Bremner, 1965). Data are presented in Table 1 .

\section{ACKNOWLEDGMENT}

This study is a part of the sedimentological survey of Leg $42 \mathrm{~A}$ samples financed by the Deutsche Forschungsgemeinschaft. I am indebted to C. Lutz, A. Schneider, and W. Schuster for laboratory work and performance of analyses.

\section{REFERENCES}

Boyce, R. E. and Bode, G. W., 1972. Carbon and carbonate analyzes, Leg 9. In Hays, J. D., et al., Initial Reports of Deep Sea Drilling Project, Volume 9: Washington (U.S. Government Printing Office), p. 797-816.

Bremner, J. M., 1965: Inorganic forms of nitrogen. In Black, C. A. (Ed.), Methods of soil analyses, Part 2, Agronomy 9, p. $1238-1255$.

Müller, J. 1977. Carbonate content, carbonate mineralogy, and bulk mineralogy of Leg 42A samples: this volume.

Sigl, W., Chamley, H., Giroud d'Argoud, G. Müller, J., and Fabricius, F., 1977. Sedimentology and environmental conditions of sapropel: this volume. 
W. SIGL

TABLE 1

Carbon and Nitrogen Analysis

\begin{tabular}{lccc}
\hline Sample (Interval in cm) & $\begin{array}{c}\text { Inorganic } \\
\text { Carbon (\%) }\end{array}$ & $\begin{array}{c}\text { Organic } \\
\text { Carbon (\%) }\end{array}$ & $\begin{array}{c}\text { Total } \\
\text { Nitrogen (\%) }\end{array}$ \\
\hline
\end{tabular}

\section{Site 371}

1-1, 100-150

4-2, 13

4-2, 15

4-2, 130

8-2, 116-118

$8, \mathrm{CC}$

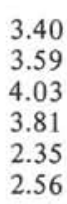

Site 372

$3-3,78-80$
$4-2,70-72$
$5-1,140$
$6-1,140-141$
$9-1,112-113 \mathrm{~A}$
$9-1,112-113 \mathrm{~B}$
$9-3,10-12$
$12-4,134-135$
44, CC

Hole 373A

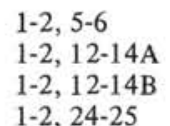

Site 374

1-1, 65-66

$1-1,123-124$

$1-1,136-138$

$1-1,144-146$

$1-2,17-19$

$1-2,145-147$

$1, \mathrm{CC}$

2-1, 57-59

2-1, 121-123

2-2, 3-4

2-2, 22-23

2-3, 21-22

2-3, 123-124

2-3, 124-125

2, CC (A)

2, CC (B)

$2, \mathrm{CC}(\mathrm{C})$

3-1, 103-104

3-1, 104-105

3-1, 105-106

3-1, 122-123

3-1, 126-127

3-1, 127-128

3-1, 129-130

3-1, 141-142

3-1, 143-144

$3, \mathrm{CC}$

4-2, 55-56

4-2, 56-57

4-2, 57-58

4-2, 58-59

4-2, 595-605

4-3, 2-4

4-3, 77-79

$4-3,79-81$

4-3, 95-97

4-3, $115-116$

4-4, 75-76

4-4, 85-86

4 , CC (A)

4, CC (B)

$\begin{array}{ll}5.17 & 6.19 \\ 4.99 & 0.20 \\ 4.28 & 0.09 \\ 2.55 & 0.09 \\ 3.27 & 0.11 \\ 4.73 & 0.12 \\ 5.02 & 0.12 \\ 6.32 & 0.35 \\ 6.86 & 0.25\end{array}$

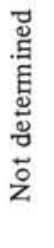

z

0.19

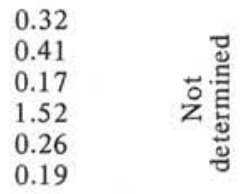

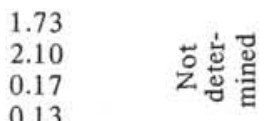

3.80

0.13

5.83

4.75

3.45

5.20

5.56

5.57

5.35

1.54

1.56

1.75

1.98

2.34

3.34

$5 . \overline{41}$

4.59

2.70

3.98

5.43

$\overline{4.14}$

4.48

4.65

$-$

0.47
0.55

2.78

1.86

4.54

4.89

5.88

3.65

2.66

3.74

3.48

2.75

2.53

3.01

3.57

4.13

3.85

\begin{tabular}{|c|c|}
\hline 0.39 & - \\
\hline 0.20 & - \\
\hline 0.31 & - \\
\hline 0.32 & - \\
\hline 0.12 & - \\
\hline 0.30 & - \\
\hline 0.28 & - \\
\hline 0.31 & - \\
\hline 0.43 & - \\
\hline 0.48 & - \\
\hline 0.60 & - \\
\hline 0.48 & - . \\
\hline 2.96 & 0.25 \\
\hline 0.42 & 0.09 \\
\hline 0.13 & - \\
\hline 1.62 & 0.14 \\
\hline 0.21 & 0.06 \\
\hline 3.21 & - \\
\hline 4.21 & 0.35 \\
\hline 0.52 & 0.08 \\
\hline 2.62 & 0.25 \\
\hline 2.52 & 0.27 \\
\hline 4.96 & 0.35 \\
\hline 0.38 & 0.10 \\
\hline 7.47 & 0.50 \\
\hline 8.72 & 0.61 \\
\hline 0.67 & 0.08 \\
\hline 0.51 & 0.10 \\
\hline 2.39 & 0.23 \\
\hline 2.48 & 0.24 \\
\hline 2.46 & 0.25 \\
\hline 0.32 & - \\
\hline 0.25 & - \\
\hline 0.18 & - \\
\hline 0.27 & - \\
\hline 0.19 & - \\
\hline 0.17 & - \\
\hline 0.14 & - \\
\hline 0.13 & - \\
\hline 0.20 & - \\
\hline 0.33 & - \\
\hline
\end{tabular}

TABLE 1 - Continued

\begin{tabular}{|c|c|c|c|}
\hline Sample (Interval in $\mathrm{cm}$ ) & $\begin{array}{c}\text { Inorganic } \\
\text { Carbon (\%) }\end{array}$ & $\begin{array}{c}\text { Organic } \\
\text { Carbon }(\%)\end{array}$ & $\begin{array}{c}\text { Total } \\
\text { Nitrogen }(\%)\end{array}$ \\
\hline $4, \mathrm{CC}(\mathrm{C})$ & 4.66 & 2.03 & 0.14 \\
\hline $4, C C$ (D) & 2.50 & 0.30 & 0.09 \\
\hline $5-1,96-97$ & 6.40 & 0.34 & - \\
\hline $5-1,138-139$ & 6.15 & 0.23 & - \\
\hline $5-2,31-32$ & - & 0.33 & 0.07 \\
\hline $5-2,32-34$ & 4.08 & 1.66 & 0.28 \\
\hline $5-2,35-36$ & 5.86 & 0.43 & 0.11 \\
\hline $5-2,39-40$ & 4.30 & 2.08 & - \\
\hline $5-2,42-43$ & - & 0.8 & 0.10 \\
\hline $5-2,47-48$ & 4.82 & 8.99 & 0.54 \\
\hline $5-2,83-84$ & 7.32 & 0.13 & - \\
\hline $5-2,121-122$ & 5.94 & 1.43 & 0.16 \\
\hline $5-2,124.5-125.5$ & 7.37 & 0.47 & 0.09 \\
\hline $5-3,9-10$ & 3.51 & 9.87 & 0.61 \\
\hline $5-3,10-11$ & 5.03 & 4.37 & 0.28 \\
\hline $5-3,48-49$ & 3.70 & 0.28 & - \\
\hline $5-3,49-50$ & 0.68 & 16.74 & 10 \\
\hline $5-3,50-51$ & 0.28 & 13.16 & 1.0 \\
\hline $5-3,51-52$ & 3.06 & 8.5 & 0.54 \\
\hline $5-3,52-53$ & 3.66 & 4.92 & - \\
\hline $5-3,53-54$ & 5.26 & 0.21 & - \\
\hline $5-3,64-66$ & 5.11 & 0.32 & - \\
\hline $5-4,24-25$ & 4.19 & 0.14 & - \\
\hline $5-4,25.7-26.5$ & 5.57 & 0.15 & - \\
\hline $5-4,28.3-29$ & 7.0 & 0.13 & - \\
\hline $5-4,87-89$ & 6.21 & 0.14 & - \\
\hline $5-5,76-78$ & 7.07 & 0.09 & - \\
\hline $5, \mathrm{CC}$ & 6.71 & 0.07 & - \\
\hline $5, \mathrm{CC}(\mathrm{A})$ & 5.94 & 0.10 & - \\
\hline $5, \mathrm{CC}(\mathrm{B})$ & 6.03 & 0.09 & - \\
\hline $6-1,4-5$ & 6.29 & 0.08 & - \\
\hline $6-1,29-30$ & 6.91 & 0.09 & - \\
\hline $6-1,40-41$ & 5.16 & 0.12 & - \\
\hline $6-1,99-101$ & 6.85 & 0.14 & - \\
\hline $6-2,40-41$ & 4.30 & 0.14 & 0.06 \\
\hline $6-2,41-42$ & 3.16 & 4.07 & 0.31 \\
\hline $6-2,42-43$ & - & 9.56 & 0.62 \\
\hline $6-2,82-83$ & 7.91 & 0.09 & - \\
\hline $6-2,97-98$ & 5.13 & 1.82 & 0.17 \\
\hline $6-2,130-131$ & 6.04 & 0.14 & - \\
\hline $6-3,7-8$ & 5.50 & 3.85 & - \\
\hline $6-3,9-9.8$ & 7.12 & 0.16 & - \\
\hline $6-3,10.9-11.7$ & 7.49 & 0.13 & - \\
\hline $6-3,12.5-13.3$ & 7.69 & 0.13 & - \\
\hline $6-3,62.5-63.2$ & 4.30 & 0.21 & - \\
\hline $6-3,63.5-64.6$ & 3.99 & 2.88 & - \\
\hline $6-3,64.6-65.8$ & 5.48 & 2.64 & 0.17 \\
\hline $6-3,65.8-66.7$ & - & 0.12 & 1.70 \\
\hline $6-3,67-68$ & - & 0.19 & 0.05 \\
\hline $6-3,124-125 \mathrm{~A}$ & 5.16 & 3.83 & 0.29 \\
\hline $6-3,124-125 B$ & 3.54 & 0.67 & 0.14 \\
\hline $6-3,125-126$ & - & 2.77 & 0.23 \\
\hline $6-4,99-100$ & 7.16 & 0.10 & - \\
\hline $6-5,40-42$ & 6.72 & 0.16 & - \\
\hline $6-5,46-48$ & 7.04 & 0.06 & - \\
\hline $6-5,57-59$ & 6.72 & 0.44 & - \\
\hline $6-5,63-65$ & 7.20 & 0.09 & - \\
\hline $6-5,67-68$ & 6.83 & 0.13 & - \\
\hline $6-5,68-69$ & 6.74 & 0.07 & - \\
\hline $6-5,69-70$ & 6.62 & 0.11 & - \\
\hline $6-5,70-71$ & 7.52 & 0.08 & - \\
\hline $6-5,110-111$ & - & 2.13 & 0.21 \\
\hline $6-6,10.7-11.3$ & 7.04 & 0.07 & - \\
\hline $6-6,11.3-12.4$ & 5.32 & 0.04 & - \\
\hline $6-6,14-15.2$ & 8.36 & 0.14 & - \\
\hline $6-6,15.2-16$ & 7.86 & 0.06 & - \\
\hline $6-6,84-86$ & 7.07 & 0.05 & - \\
\hline $6, \mathrm{CC}(\mathrm{A})$ & 7.42 & 0.04 & - \\
\hline 6, CC (B) & 7.14 & 0.06 & - \\
\hline
\end{tabular}


TABLE 1 - Continued

\begin{tabular}{|c|c|c|c|}
\hline Sample (Interval in $\mathrm{cm}$ ) & $\begin{array}{c}\text { Inorganic } \\
\text { Carbon (\%) }\end{array}$ & $\begin{array}{c}\text { Organic } \\
\text { Carbon (\%) }\end{array}$ & $\begin{array}{c}\text { Total } \\
\text { Nitrogen }(\%)\end{array}$ \\
\hline $7-1,83-88$ & 5.55 & 0.09 & - \\
\hline $7-2,68-73$ & 5.54 & 0.12 & - \\
\hline $7-3,13-18$ & 7.07 & 0.08 & - \\
\hline $7-3,72-77$ & 5.23 & 0.24 & - \\
\hline $7-4,83-88$ & 4.90 & - & - \\
\hline $7-5,53-58$ & 7.19 & 0.06 & - \\
\hline $7-5,71-76$ & 6.22 & 0.19 & - \\
\hline $7-6,52-57$ & 6.53 & 0.12 & - \\
\hline $8-1,115-116$ & 8.12 & 0.07 & - \\
\hline $8-2,24-26$ & 6.28 & 0.03 & - \\
\hline $8-2,63-64$ & 7.53 & 0.06 & - \\
\hline $8-3,98-99$ & 7.52 & 0.07 & - \\
\hline $9-1,150-151$ & 6.35 & 1.33 & - \\
\hline $9-2,1-2$ & - & 1.93 & 0.17 \\
\hline $9-3,13-14$ & - & 2.31 & 0.19 \\
\hline $9-3,15-16$ & 7.61 & 1.17 & - \\
\hline $9-3,72-77$ & 8.08 & 0.15 & - \\
\hline $11-2,112$ & 10.34 & 0.13 & - \\
\hline $11-2,120-121$ & 9.35 & 0.09 & - \\
\hline $11-2,147-149$ & 9.0 & 0.72 & 0.09 \\
\hline $11, \mathrm{CC}(\mathrm{A})$ & 8.21 & 1.44 & - \\
\hline $11, \mathrm{CC}(\mathrm{B})$ & 9.21 & 1.73 & 0.18 \\
\hline $11, \mathrm{CC}(\mathrm{C})$ & 8.31 & 0.57 & - \\
\hline $12-2,99-100$ & 2.48 & 0.37 & - \\
\hline $13-1,133-135$ & 2.35 & 0.37 & - \\
\hline $14-1,40-42$ & - & 0.75 & 0.08 \\
\hline $14-1$, TOP & 2.49 & 0.65 & - \\
\hline $14-1,100-102$ & 2.28 & 0.43 & - \\
\hline $14-2,35-37$ & - & 0.51 & 0.11 \\
\hline $15-1,69-70$ & 2.99 & 0.55 & - \\
\hline $15-1,100-102$ & 2.90 & 0.41 & - \\
\hline $15-2,79-81$ & 2.77 & 0.30 & - \\
\hline $16-1,84-87$ & 4.80 & 2.47 & - \\
\hline $17-1,62-63$ & 1.89 & 2.16 & - \\
\hline $17-1,67-68$ & 4.76 & 4.76 & - \\
\hline $17-1,72-75$ & - & 4.80 & 0.18 \\
\hline $17-1,80-81$ & 4.44 & 2.03 & - \\
\hline $19-1,28-29$ & 6.14 & 0.22 & - \\
\hline $19-1,52-53$ & 3.34 & 0.41 & - \\
\hline $20-1,24-25$ & 1.31 & 5.32 & 0.25 \\
\hline $21-1,105-108$ & 2.68 & 0.88 & - \\
\hline $25, \mathrm{CC}$ & 8.56 & 0.18 & - \\
\hline $26, \mathrm{CC}$ & 7.63 & 0.09 & - \\
\hline \multicolumn{4}{|l|}{ Site 375} \\
\hline $1-1,50-51$ & 4.45 & 0.12 & - \\
\hline $1-1,123-125$ & 3.02 & 0.05 & - \\
\hline $2-2,39-40$ & 2.70 & 0.09 & - \\
\hline $2-3,65-66$ & 1.67 & 0.09 & - \\
\hline $2-3,96-100$ & 1.76 & 0.08 & - \\
\hline $2-3,144-150$ & 5.24 & 0.10 & - \\
\hline $2-4,23-24$ & 3.50 & 0.05 & - \\
\hline $4-1,26-27$ & 4.08 & 0.16 & - \\
\hline $4-2,70-71$ & 4.88 & 0.17 & - \\
\hline $4-3,79-80$ & 3.26 & 0.14 & - \\
\hline $4-4,37-38$ & 4.40 & 0.31 & - \\
\hline $4-4,39-40$ & 4.21 & 0.30 & - \\
\hline $4-4,40-40.5$ & 1.42 & 1.01 & - \\
\hline $4-4,40.5-42$ & 2.43 & 4.47 & 0.26 \\
\hline $4-4,42-43$ & 3.80 & 5.57 & - \\
\hline $4-4,43-44$ & 4.20 & 6.37 & 0.29 \\
\hline $4-4,44-45$ & 6.26 & 3.65 & - \\
\hline $4-4,45-46$ & 1.08 & 3.65 & - \\
\hline $4-4,46-47$ & 4.13 & 5.74 & 0.27 \\
\hline $4-4,47-48$ & 5.42 & 5.79 & - \\
\hline $4-4,48-49$ & 6.03 & 7.02 & - \\
\hline $4-4,49-50$ & 4.34 & 4.70 & - \\
\hline $4-4,50-51$ & 4.98 & 5.40 & 0.26 \\
\hline
\end{tabular}

TABLE 1 - Continued

\begin{tabular}{|c|c|c|c|}
\hline Sample (Interval in cm) & $\begin{array}{l}\text { Inorganic } \\
\text { Carbon (\%) }\end{array}$ & $\begin{array}{c}\text { Organic } \\
\text { Carbon (\%) }\end{array}$ & $\begin{array}{c}\text { Total } \\
\text { Nitrogen }(\%)\end{array}$ \\
\hline $4-4,51-52$ & 5.47 & 6.29 & - \\
\hline $4-4,52-52.5$ & 5.59 & 6.15 & - \\
\hline $4-4,53-54$ & 4.11 & 6.77 & - \\
\hline $4-4,54-55$ & 4.30 & 6.81 & - \\
\hline $4-4,55-56$ & 5.79 & 6.15 & - \\
\hline $4-4,56-57$ & 5.90 & 6.20 & 0.31 \\
\hline $4-4,57-58$ & 6.83 & 4.15 & - \\
\hline $4-4,58-58.5$ & 8.22 & 3.40 & - \\
\hline $4-4,59-61$ & 5.03 & 4.72 & - \\
\hline $4-4,61-62$ & 0.83 & 3.87 & - \\
\hline $4-4,62-63$ & 3.58 & 0.93 & - \\
\hline $4-4,63-64$ & 3.61 & 0.92 & 0.06 \\
\hline $4-5,88-89$ & 3.31 & 0.28 & - \\
\hline $4, \mathrm{CC}$ & 5.52 & 0.29 & - \\
\hline $5-1,74-75$ & 5.65 & 0.16 & - \\
\hline $5-1,81-83$ & 6.62 & 0.39 & - \\
\hline $5-3,96-97$ & 1.44 & 0.17 & - \\
\hline $5-5,36-38$ & 4.69 & 0.34 & - \\
\hline $5-5,47-48$ & 4.20 & 0.46 & - \\
\hline $5-5,139-141$ & 2.09 & 3.72 & 0.23 \\
\hline $5, \mathrm{CC}$ & 0.61 & 3.35 & - \\
\hline $6-2,105-106$ & 2.71 & 0.16 & - \\
\hline $6-3,53-55$ & 2.60 & 2.18 & - \\
\hline $6-3,91-92$ & 3.08 & 0.33 & - \\
\hline $6-5,48-50$ & 3.12 & 0.54 & - \\
\hline $6, \mathrm{CC}$ & 2.67 & 0.51 & - \\
\hline $7-1,51-52$ & 5.11 & 0.32 & - \\
\hline $7-3,46-47$ & 2.70 & 0.26 & - \\
\hline $7-5,51-52$ & 3.53 & 0.51 & - \\
\hline $7, \mathrm{CC}$ & 4.88 & 0.16 & - \\
\hline $8-1,83-84$ & 5.91 & 0.15 & - \\
\hline $8-1,103-104$ & 5.71 & 0.12 & - \\
\hline $8-3,39-40$ & 1.52 & 0.21 & - \\
\hline $8-3,90-91$ & 5.32 & 0.11 & - \\
\hline $8-4,109-110$ & 5.41 & 0.09 & - \\
\hline $8-6,93-94$ & 1.13 & 0.13 & - \\
\hline $8, \mathrm{CC}$ & 4.95 & 0.13 & - \\
\hline $9-1,36-37$ & 2.82 & 0.27 & - \\
\hline $9-3,41-42$ & 1.07 & 0.68 & - \\
\hline $9-6,63-69$ & 2.74 & 0.14 & - \\
\hline 9, CC & 4.51 & 0.16 & - \\
\hline $10-2,22-24$ & 5.16 & 0.07 & - \\
\hline $10-2,24-25$ & 6.35 & 0.07 & - \\
\hline $11-1,132-134$ & 7.31 & 0.04 & - \\
\hline $11-2,55-57$ & 5.49 & 0.07 & - \\
\hline $11-2,157-158$ & 6.29 & 0.09 & - \\
\hline $12, \mathrm{CC}$ & 6.49 & 0.09 & - \\
\hline $13, \mathrm{CC}$ & 0.96 & 0.41 & - \\
\hline \multicolumn{4}{|l|}{ Site 376} \\
\hline $1-1,38-39$ & 4.88 & 0.14 & - \\
\hline $1-1,124-125$ & 3.82 & 0.13 & - \\
\hline $1-2,115-116$ & 4.22 & 2.78 & - \\
\hline $1-3,10-12$ & 3.47 & 0.48 & - \\
\hline $1-3,13-15$ & 4.37 & 1.36 & 6.11 \\
\hline $1-3,18-20$ & 4.21 & 2.09 & - \\
\hline $1-3,24-26$ & 4.63 & 2.09 & 0.17 \\
\hline $1-3,31-33$ & 4.27 & 2.21 & - \\
\hline $1-4,65-66$ & 3.59 & 5.55 & 0.42 \\
\hline $1-4,88-89$ & 3.72 & 2.64 & - \\
\hline $1-5,119-120$ & 3.62 & 0.13 & - \\
\hline $1, \mathrm{CC}$ & 2.82 & 0.09 & - \\
\hline $2-1,87-88$ & 4.70 & 0.15 & - \\
\hline $2-2,144-145$ & 4.60 & 5.28 & 0.3 \\
\hline $2-3,0-1$ & 3.73 & 1.04 & - \\
\hline $2-3,8-9$ & 4.48 & 2.70 & - \\
\hline $2-3,147-148$ & 5.27 & 1.90 & - \\
\hline $2-4,30-31$ & 6.70 & 2.32 & - \\
\hline $2-4,54-55$ & 5.02 & 0.23 & - \\
\hline
\end{tabular}


TABLE 1 - Continued

\begin{tabular}{|c|c|c|c|}
\hline Sample (Interval in $\mathrm{cm}$ ) & $\begin{array}{l}\text { Inorganic } \\
\text { Carbon (\%) }\end{array}$ & $\begin{array}{c}\text { Organic } \\
\text { Carbon (\%) }\end{array}$ & $\begin{array}{c}\text { Total } \\
\text { Nitrogen }(\%)\end{array}$ \\
\hline $2, \mathrm{CC}$ & 4.11 & 0.13 & - \\
\hline $3-1,135-136$ & 3.65 & 0.20 & - \\
\hline $3-2,38-39$ & 5.12 & 3.44 & - \\
\hline $3-2,89-90$ & 5.71 & 0.40 & - \\
\hline $3-2,115-116$ & 5.17 & 0.37 & - \\
\hline $3-3,79$ & 4.32 & 0.15 & - \\
\hline $3-5,137-138$ & 6.51 & 0.10 & - \\
\hline $3, \mathrm{CC}$ & 5.29 & 0.07 & - \\
\hline $4-1,80-81$ & 4.59 & 0.13 & - \\
\hline $4, \mathrm{CC}$ & 4.49 & 0.09 & - \\
\hline $5-1,103-104$ & 4.35 & 0.19 & - \\
\hline $5-2,72-73$ & 4.48 & 0.10 & - \\
\hline $5-2,115-116$ & 3.79 & 4.56 & 0.22 \\
\hline $5-3,60-61$ & 6.17 & 0.11 & - \\
\hline $5-4,38-39$ & 5.73 & 0.09 & - \\
\hline $5-4,64-65$ & 5.13 & 0.53 & - \\
\hline $5-4,105-106$ & 3.31 & 8.92 & - \\
\hline $5-5,95-96$ & 3.35 & 0.21 & - \\
\hline $5, \mathrm{CC}$ & 4.52 & 0.11 & - \\
\hline $6-1,133-134$ & 4.34 & 0.11 & - \\
\hline $6-2,83-84$ & 5.09 & 0.08 & - \\
\hline $6-3,94-95$ & 1.06 & 1.23 & 0.09 \\
\hline $6-4,50-51$ & 7.73 & 0.12 & - \\
\hline $6-4,56-57$ & 6.90 & 0.28 & - \\
\hline $6-4,57-58$ & 7.04 & 0.33 & - \\
\hline $6-4,58-59$ & 7.68 & 0.74 & - \\
\hline $6-4,60-61$ & 7.64 & 0.99 & 0.09 \\
\hline $6-4,61-62$ & 8.27 & 1.18 & - \\
\hline $6-4,63-64$ & 6.18 & 3.75 & 0.17 \\
\hline $6-4,64-65$ & 6.86 & 2.11 & - \\
\hline $6-4,66-67$ & 6.14 & 2.16 & 0.11 \\
\hline $6-4,68-69$ & 6.05 & 3.44 & - \\
\hline $6-4,70-71$ & 7.22 & 1.60 & - \\
\hline $6-4,72-73$ & 7.44 & 1.33 & - \\
\hline $6-4,74-75$ & 9.18 & 1.27 & 0.09 \\
\hline $6-4,75-76$ & 8.16 & 0.80 & - \\
\hline $6-4,77-78$ & 7.65 & 0.83 & - \\
\hline $6-4,78-79$ & 7.14 & 0.68 & - \\
\hline $6-4,81-82$ & 7.45 & 0.21 & - \\
\hline $7-1,59-60$ & 3.66 & 0.11 & - \\
\hline $7-2,51-52$ & 3.70 & 0.16 & - \\
\hline $7, \mathrm{CC}$ & 4.31 & 0.17 & - \\
\hline $8-1,140-141$ & 3.77 & 0.25 & - \\
\hline $8-2,69-70$ & 5.09 & 0.17 & - \\
\hline $8-3,17-18$ & 4.04 & 0.24 & - \\
\hline $8-3,31-32$ & 3.96 & 0.15 & - \\
\hline $8-3,36-37$ & 3.52 & 0.11 & - \\
\hline $8, \mathrm{CC}$ & 4.42 & 0.12 & - \\
\hline $9-1,50-51$ & 4.25 & 0.18 & - \\
\hline $9-1,81-83$ & 4.32 & 0.60 & - \\
\hline $9-2,70-71$ & 3.55 & 0.29 & - \\
\hline $9-3,70-71$ & 3.54 & 0.24 & - \\
\hline $9-3,39-40$ & - & 0.60 & - \\
\hline $9-4,4-5$ & 4.48 & 0.14 & - \\
\hline $9-4,80-81$ & 4.79 & 0.07 & - \\
\hline $9, \mathrm{CC}$ & 3.44 & 0.19 & - \\
\hline $10-1,101-102$ & 4.82 & 0.13 & - \\
\hline $10-3,100-101$ & 4.27 & 0.24 & - \\
\hline $10, \mathrm{CC}$ & 4.49 & 0.16 & - \\
\hline $11-1,139-140$ & 4.76 & 0.19 & - \\
\hline $11-2,130-131$ & 8.56 & 0.75 & - \\
\hline $11-2,133-136$ & 5.37 & 1.22 & - \\
\hline $11, \mathrm{CC}$ & 8.38 & 0.14 & - \\
\hline $12-1,22-28$ & 7.17 & 0.64 & - \\
\hline $12-1,40-42$ & 4.49 & 0.55 & - \\
\hline $12-2,49-51$ & 4.33 & 0.77 & - \\
\hline $12-2,70-71$ & 2.77 & 0.26 & - \\
\hline $12-2,90-91$ & 4.47 & 0.42 & - \\
\hline
\end{tabular}

TABLE 1 - Continued

\begin{tabular}{lccc}
\hline Sample (Interval in cm) & $\begin{array}{c}\text { Inorganic } \\
\text { Carbon }(\%)\end{array}$ & $\begin{array}{c}\text { Organic } \\
\text { Carbon (\%) }\end{array}$ & $\begin{array}{c}\text { Total } \\
\text { Nitrogen (\%) }\end{array}$ \\
\hline $12-3,130-131$ & 6.72 & 0.38 & - \\
$12-4,70-71$ & 3.28 & 0.30 & - \\
$12-5,130-131$ & 4.52 & 0.24 & - \\
12, CC & 4.70 & 0.11 & - \\
$13-1,114-115$ & 7.90 & 0.15 & - \\
$13-2,71-72$ & 3.79 & 0.23 & - \\
$13-4,101-102$ & 3.00 & 0.28 & - \\
13, CC & 2.47 & 0.22 & - \\
14, CC & 2.85 & 0.24 & - \\
$15-1,137-138$ & 5.43 & 0.11 & - \\
$15-2,35-36$ & 5.61 & 0.17 & - \\
$15-2,125-126$ & 4.34 & 0.21 & - \\
$15-3,2-3$ & 5.34 & 0.15 & - \\
15, CC & 4.89 & 0.12 & - \\
$17-1,45-47$ & 4.43 & 0.13 & -
\end{tabular}

Site 377

$1-1,65-67$
$1-1,104-106$
$1-1,149-150$
$1-2,10-11$
$1-2,28-29$
$1-2,97-98$
$1-2,109-111$
$2-1,105-106$
$3-2,19-23$
$4-3,140-142$

6.10

0.29

8.21

5.99

4.02

4.48

5.47

5.98

6.21

2.81

1.50

0.16

2.91

4.06

3.47

3.47

0.32

0.42

0.41
1.04

Hole 378

\begin{tabular}{llll}
$1-1,133-134$ & 6.59 & 7.15 & - \\
$1-2,64-65 \mathrm{~A}$ & 5.49 & 3.11 & - \\
$1-2,64-65 \mathrm{~B}$ & 4.93 & 5.88 & - \\
$1-2,65-66$ & 5.22 & 6.98 & - \\
$1-2,66-67$ & 3.97 & 6.93 & - \\
$1-2,67-68$ & 5.85 & 6.35 & - \\
$1-2,69-70$ & 5.75 & 6.98 & - \\
$1-2,71-72$ & 7.43 & 0.40 & - \\
$3-3,122-123$ & 4.21 & 4.08 & - \\
$6-2,18-19$ & 4.43 & 5.16 & - \\
$6-3,84-85$ & 5.70 & 4.63 & - \\
$6-3,95-96$ & 6.36 & 5.50 & - \\
$7-4,71-72$ & 4.51 & 3.22 & - \\
$8-1,124-125$ & 6.00 & 3.76 & - \\
$8-2,2-3$ & 4.11 & 0.75 & - \\
$8-2,13-14$ & 4.11 & 3.32 & - \\
$8-2,31-35$ & 3.94 & 3.96 & - \\
$8-2,45-46$ & 3.27 & 5.17 & - \\
$8-2,47-48$ & 4.22 & 3.05 & - \\
$8-2,59-60$ & 2.86 & 0.29 & - \\
$8-2,70-71$ & 5.21 & 0.36 & - \\
$11-1,148-149$ & 4.97 & 1.52 & - \\
$11-2,1-2$ & 4.56 & 4.16 & - \\
$11-2,28-29$ & 4.84 & 1.69 & - \\
$11-2,73-74$ & 6.43 & 0.34 & - \\
$11-2,98-99$ & 5.01 & 1.13 & - \\
$11-2,136-137$ & 5.45 & 1.51 & - \\
$11-4,9-10$ & 2.91 & 1.64 & - \\
$11-4,22-23$ & 4.06 & 1.83 & - \\
$11-4,37-38$ & 3.76 & 1.75 & - \\
$11-4,48-49$ & 3.28 & 1.66 & - \\
$11-4,64-65$ & 3.95 & 2.10 & - \\
$11-4,130-131$ & 3.56 & 1.95 & - \\
\hline 103784 & & & -
\end{tabular}

Hole 378A
1-2, 20-21
$1-3,14-15$
$1-3,143-145$

$1-5,85-86$

$\begin{array}{lll}4.20 & 2.43 & - \\ 5.20 & 1.72 & - \\ 5.94 & 0.42 & - \\ 6.53 & 2.39 & -\end{array}$

